\title{
Urothelial Cell
}

National Cancer Institute

\section{Source}

National Cancer Institute. Urothelial Cell. NCI Thesaurus. Code C33841.

A cell found in the layer of transitional epithelium in the wall of the bladder, ureter, and renal pelvis, external to the lamina propria. 